\title{
Drei Dimensionen der Verantwortung von Konsumenten und Unternehmen ${ }^{*}$
}

\section{Christian NEUHÄUSER ${ }^{*}$}

Wenn Konsumethik eine nachhaltige Wirkung entfalten soll, dann muss sie als Konsumentenverantwortung verstanden und zur Unternehmensverantwortung ins Verhältnis gesetzt werden. Dies bringt zwei entscheidende Vorteile: Erstens lässt sich die zentrale Rolle der moralischen Verantwortung von Konsumenten und Unternehmen herausarbeiten und ihre Notwendigkeit für eine faire und ökologische Transformation der politischen und rechtlichen Verantwortung begründen. Zweitens lässt sich zeigen, wie genau Konsumenten- und Unternehmensverantwortung miteinander verschränkt sind und warum sie wechselseitig aufeinander angewiesen sind, um ihre volle Wirkung zu entfalten. Damit lassen sich auch zwei der grundlegenden Kritiken der Konsumenten- und Unternehmensverantwortung, nämlich dass sie wirkungslos und normativ unbegründet seien, aus dem Weg räumen.

Schlagwörter: Konsumethik, Konsumentenverantwortung, Unternehmensethik, Unternehmensverantwortung, politische Verantwortung, moralische Verantwortung

\section{Three Dimensions of the Responsibility of Consumers and Corporations}

Consumer ethics needs to focus on the responsibility of consumers and be put in touch with corporate responsibility in order to be meaningful. This approach has two crucial advantages: First the central role of a moral responsibility of consumers and corporations for a transformation of their political and legal responsibility in the direction of more fairness and sustainability becomes clearly visible. Second it is possible to see how consumer and corporate responsibility relate to each other and how, if taken together, their impact will grow considerably. Conceptualizing consumer ethics as consumer responsibility thereby also belps to refute two common critical arguments against consumer etbics, namely that it is unfounded and without any considerable effect.

Keywords: Consumer ethics, consumer responsibility, corporate ethics, corporate responsibility, political responsibility, moral responsibility

Bewusster Konsum, dem es um mehr als nur ein rein ökonomisches Preis-LeistungsVerhältnis geht, verfügt bereits über eine längere Tradition und ist inzwischen in eine

Beitrag eingereicht am 25.02.2012; nach doppelt verdecktem Gutachterverfahren überarbeitete Fassung angenommen am 10.10.2012.

Dr. Christian Neuhäuser, Universität Luzern, Kultur- und Sozialwissenschaftliche Fakultät, Frohburgstr. 3, Postfach 4466, CH-6002 Luzern, Tel: +41-(0)41-229-5620, E-Mail: christian.neuhaeuser@unilu.ch, Forschungsschwerpunkte: Wirtschaftsethik, Handlungstheorie, Menschenwürde und Menschenrechte, Globale Gerechtigkeit, Umweltgerechtigkeit. 
dritte, nämlich ethische Phase eingetreten (vgl. Busse 2006; Ullrich 2007; König 2008). Zunächst haben Konsumenten in der Wohlstandsphase der 60er Jahre des letzten Jahrhunderts ihren Konsum ästhetisiert. Dabei ging es nicht mehr allein um günstige Preise und große Funktionalität, sondern zunehmend um Geschmack, Design und Mode. Dann kam seit den späten 80er Jahren ein gesundheitlicher Aspekt hinzu. Insbesondere Lebensmittel mussten nicht nur gut schmecken, sondern auch gesund sein. Dies war der Auslöser der Biobewegung, die seit den 90er Jahren und bis heute stetig wächst. Biolebensmittel versprechen weniger schädlich zu sein und mehr Vitamine und Nährstoffe zu enthalten, also genau jene Gesundheitsaspekte, die im Konsum zunehmend nachgefragt werden. Etwas Ähnliches gilt beispielsweise für unbehandelte Möbel und Bekleidung aus natürlichen Fasern.

Aus dieser zunächst auf die eigenen Gesundheitsbedürfnisse konzentrierten Biobewegung heraus hat sich ein ethischer Konsum entwickelt. Denn inzwischen spielen beim Biokonsum auch der Umgang mit Tieren und der Erhalt der Natur eine zentrale Rolle. Wegen des großen Erfolgs dieser tier- und umweltethischen Konsumbewegung ist zudem der faire Konsum aus der sehr kleinen Nische der Eine-Welt-Läden herausgetreten und erobert einen wachsenden Marktanteil. ${ }^{1}$ Welche Zukunft wird dieser ethische Konsum haben? Wird er das Konsumverhalten nachhaltig und flächendeckend reformieren und gewissermaßen eine friedliche Revolution der angeblich moralbefreiten Märkte in Gang setzen? Oder hat er sein Potential schon weitestgehend ausgeschöpft und bleibt ein relativ kleines Marktsegment für Besserverdienende, die sich um ihre Gesinnung sorgen?

Um diese Frage überhaupt angemessen angehen zu können, muss meiner Einschätzung nach Konsumethik vor allem als Konsumentenverantwortung verstanden und zur Unternehmensverantwortung ins Verhältnis gesetzt werden. ${ }^{2}$ Nur als Konsumentenverantwortung kann Konsumethik eine größere Breitenwirkung entfalten (vgl. Heidbrink/Schmidt/Ahaus 2011; Heidbrink/Schmidt 2011). Denn nur dann verstehen Konsumenten ihren ethischen Konsum nicht als freiwillige und großzügige Mehrleistung, sondern sie sehen sich tatsächlich dazu verpflichtet, ethisch zu konsumieren, weil sie in einer entsprechenden Verantwortung stehen. Und nur wenn diese Konsumentenverantwortung in ein angemessenes Verhältnis zur Unternehmensverantwortung gesetzt wird, hat der Funke des ethischen Konsums eine in der Praxis realistische

\footnotetext{
$1 \quad$ Im Jahre 1996 wurden in Deutschland Produkte mit Fair-Trade-Siegel im Wert von 52 Mio. Euro verkauft, im Jahre 2011 im Wert von 400 Mio. Euro. Das entspricht einer Umsatzsteigerung von 800 Prozent in 15 Jahren. Vgl. http://www.fairtrade-deutschland.de/produkte/absatz-fairtradeprodukte/ (zuletzt abgerufen am 27.02.2012).

2 Damit ist nicht gemeint, dass Konsumethik vollständig in einer Konsumentenverantwortung als reine Verantwortungsethik im Sinne von Max Weber aufgeht, sondern nur, dass Konsumentenverantwortung als zentrales Moment der Konsumethik zu verstehen ist. Eine gute Übersicht über Fragen der Unternehmensethik liefern Crane und Matten (2010: 51ff.). Für zwei gegenwärtige grundsätzliche Auseinandersetzungen: Hübscher (2011), Neuhäuser (2011a).
} 
Chance, von wenigen plakativen Produkten auf das gesamte Marktgeschehen überzuspringen. ${ }^{3}$

Ich möchte diese Thesen verteidigen, indem ich drei Dimensionen des Verantwortungsbegriffs unterscheide und auf das Verhältnis von Konsumenten und Unternehmen anwende. Dabei handelt es sich um die rechtliche, die politische und die moralische Dimension der Verantwortung. Obwohl die rechtliche und die politische Dimension jeweils eine wichtige Eigenständigkeit besitzen, ist doch die moralische Dimension zentral. Von ihr geht der entscheidende Reformimpuls, nicht nur für Konsumenten, sondern auch für Unternehmen aus und sie verschafft der politischen und rechtlichen Verantwortung eine zusätzliche und dringend benötigte Tiefe. Nur wenn zunehmend viele Konsumenten sich selbst als aus moralischen Gründen Handelnde verstehen und nur wenn sie Unternehmen als moralische Akteure adressieren, kann sich der ethische Konsum flächendeckend ausbreiten und eine steigende politische und rechtliche Wirkung entfalten, so die Idee.

Es ist wichtig zu betonen, dass es sich bei diesen drei Dimensionen der Verantwortung nicht um klar voneinander abgegrenzte Handlungsräume oder Handlungsformen handelt. Vielmehr können im Konsum und unternehmerischem Handeln alle drei Formen zugleich auftreten. Es handelt sich also um eine analytische Unterscheidung dreier normativer Standpunkte. Außerdem ist diese Unterscheidung idealtypisch, weil die meisten Akteure keinen dieser Standpunkte in seiner Reinform einnehmen. Das soll hier auch gar nicht behauptet werden. Vielmehr geht es gerade darum herauszuarbeiten, dass Konsumenten und Unternehmen auch politische und rechtliche Prozesse aus moralischer Perspektive betrachten. Insbesondere die moralische Dimension politischen Handelns wird dabei eine wichtige Rolle spielen. Es handelt sich also um drei in der Praxis miteinander verschränkte Dimensionen normativer Verantwortung, die hier für analytische Zwecke klar unterschieden werden.

Die folgende Argumentation besteht aus vier Schritten. In einem ersten vorbereitenden Schritt wird der Verantwortungsbegriff analysiert. In einem zweiten Schritt wird die rechtliche und politische Dimension der Verantwortung von Konsumenten und Unternehmen diskutiert und in einem dritten Schritt die zentrale Stellung der moralischen Verantwortung hervorgehoben. In einem vierten und letzten Schritt wird die enge Verschränkung von Konsumenten- und Unternehmensverantwortung besonders in ihrer moralischen und politischen Dimension herausgestellt. Damit soll nicht gesagt sein, dass die rechtliche Dimension und die damit verbundene Rahmenordnung für Märkte keine Rolle spielt. Vielmehr geht es auch und nicht zuletzt darum, deren moralische und politische Voraussetzungen hervorzuheben.

\section{Verantwortung als vierstellige Relation und drei normative Dimensionen}

Verantwortung lässt sich allgemein als vierstellige Relation analysieren; dies gilt auch für Konsumenten- und Unternehmensverantwortung. Damit ist gemeint, dass voll-

3 Es gibt eine Reihe von Skeptikern die der Meinung sind, dass ethischer oder kritischer Konsum immer eine Nischenerscheinung bleiben wird, bzw. zunehmend vereinnahmt wird: Hartmann (2009), Heath/Potter (2009), Devinney/Auger/Eckhardt (2010). 
ständige Sätze der Zuschreibung von Verantwortung grundsätzlich diese Form haben: Urheber (U) ist gegenüber Adressat (A) für Ereignis (E) auf Grundlage des Maßstabes (M) verantwortlich (vgl. Lohmann 2002; Heidbrink 2003; Bayertz 1995). Diese Bestimmung fällt so allgemein aus, damit möglichst viele der üblichen Arten über Verantwortung zu sprechen, auch berücksichtigt werden können. Dazu gehört selbst die Rede von Verantwortung als kausale Verursachung, insbesondere im Bereich von Naturereignissen. In diesem Sinne wird beispielsweise das Unwetter für die Entwurzelung zahlreicher Bäume umgangssprachlich „,verantwortlich“ gemacht. Alle vier Relationen sind natürlich erklärungsbedürftig und dies soll hier recht kurz, aber bereits mit einem Blick auf Konsumenten- und Unternehmensverantwortung, geschehen. ${ }^{4}$

Der Urheber ist stets derjenige, der das fragliche Ereignis zu verantworten hat; in dem eben genannten Beispiel war das ein Unwetter. Wenn es jedoch um rechtliche, politische und moralische Verantwortung geht, dann können nur intentional und frei handelnde Akteure verantwortlich sein. Denn nur diese Akteure bringen die notwendigen Voraussetzungen mit, um auf normative Weise verantwortlich zu sein. Nur sie sind in der Lage, den rechtlichen, politischen oder moralischen Standpunkt einzunehmen und ihr Handeln selbstständig danach zu bestimmen (vgl. Wallace 2006: 144-164). Konsumenten und Unternehmen, als die verantwortlichen Akteure, um die es hier geht, müssten also diese Voraussetzungen mitbringen. Bei individuellen Konsumenten stellt diese Annahme keine besondere Schwierigkeit dar, weil Menschen normalerweise als grundsätzlich freie Akteure angesehen werden. Fraglich ist allerdings, ob Unternehmen solche intentional handelnde, freie und verantwortungsfähige Akteure sind. Ich werde hier jedoch nicht speziell dafür argumentieren, sondern dies auf Grundlage bestehender Argumentationen als gegeben voraussetzen. ${ }^{5}$

Um bestimmen zu können, wer die Adressaten der Konsumenten- und Unternehmensverantwortung sind und für welche Ereignisse diese Verantwortlichkeiten bestehen, muss zugleich der Maßstab geklärt sein. Für rechtliche Verantwortung gibt das positiv geltende Recht den Maßstab vor. Der Adressat ist dann die Rechtsgemeinschaft bzw. der Staat als Garant der Rechtsordnung. Verantwortliche Akteure haben demnach die rechtliche Verantwortung, sich an die geltenden Gesetze zu halten. Für politische Verantwortung liegt kein derart kodifizierter Maßstab vor. Vielmehr sind es die nur implizit politisch legitimierten Normen, die den Maßstab vorgeben. Welche Normen politisch legitimiert sind, das wird immer wieder neu auf prozedurale Weise von der politischen Gemeinschaft und in der politischen Öffentlichkeit ausgehandelt. ${ }^{6}$ Ver-

4 Eine ausführlichere Darstellung meiner Auffassung dieser vier Relationen findet sich in Neuhäuser (2011b). Kurz zuvor hatte ich noch mit nur drei Relationen gearbeitet und die Relevanz des normativen Maßstabs unterschätzt (vgl. Neuhäuser 2011a: 37).

5 Bekannte Ansätze stammen von French (1979, 1995) und List/Pettit (2011: 175ff.). Ich selbst habe in einer in vielen Teilen zustimmender, aber in mancher Hinsicht kritischer Absetzung von diesen Positionen ausführlich für die Verantwortungsfähigkeit von Unternehmen argumentiert in Neuhäuser (2011a).

6 Vgl. für dieses Verständnis von politischer Legitimität die Diskussion von Peter (2007). Die Art und Weise wie hier zwischen Recht, Politik und Moral unterschieden wird, folgt im Wesentlichen Karl Jaspers (2012), auf eine Diskussion der existentialistischen Dimension der metaphysischen Schuld von Jaspers wird demgegenüber verzichtet. 
antwortliche Akteure haben dieser politischen Gemeinschaft gegenüber eine politische Verantwortung, sich an die aktuell legitimen Normen zu halten, können aber zugleich auf einen politischen Wandel drängen.

Der Maßstab für moralische Verantwortung sind moralische Normen. Moralische Verantwortung besteht gegenüber der moralischen Gemeinschaft und sie besteht dafür, sein Handeln an moralischen Regeln auszurichten. Die besondere Schwierigkeit der moralischen Verantwortung scheint darin zu bestehen, dass moralische Normen kaum greifbar sind. Es gibt weder kodifizierte Normen wie im Recht, noch etablierte Verfahren zur Normgewinnung wie in der Politik. Moralphilosophen berufen sich gerne auf die rational feststellbare Objektivität moralischer Normen. Das Problem dabei ist allerdings, dass selbst sie zu sehr unterschiedlichen Ergebnissen kommen. ${ }^{7}$ So wirkt es auf den ersten Blick, als sei eine moralische Verantwortung als Maßstab für das Handeln von Konsumenten und Unternehmen äußerst ungeeignet, ja geradezu wertlos, weil verschiedene Gruppen stets unterschiedliche und bloß kontingente Vorstellungen von Moral besitzen, die andere nicht teilen (vgl. Waldron 1999: 176-187; Appiah 2007: 69-94).

Trotz der fehlenden Letztbegründbarkeit moralischer Ansprüche ist eine moralische Konsumenten- und Unternehmensverantwortung keineswegs sinnlos. Denn tatsächlich gibt es ein minimalethisches Verfahren, um mit dem Problem des moralischen Pluralismus umzugehen. Dies nimmt die moralische Grundhaltung aller betroffenen Akteure zum Ausgangspunkt, um dann über das Kohärenzverfahren des Überlegungsgleichgewichts zu einem pragmatischen Konsens zu gelangen. ${ }^{8}$ Die Akteure reflektieren über ihre moralische Haltung angesichts der wohlerwogenen und trotzdem abweichenden Position anderer Akteure, um dabei zu einer möglichst großen Übereinstimmung zu gelangen. ${ }^{9}$ Diese moralische Reflexion geschieht nicht nur in einem privaten, sondern auch in einem öffentlichen und zivilgesellschaftlichen Raum. Es handelt sich also um einen öffentlichen moralischen Diskurs mit Begründungs-, aber ohne Letztbegründungsanspruch. ${ }^{10}$ Tatsächlich kann man die Entwicklung der

$7 \quad$ Dies stellt Wallace (2011) in seiner Auseinandersetzung mit verschiedenen Positionen der realistischen, konstruktivistischen und emotivistischen Begründung von Normen heraus.

8 Die Idee des Überlegungsgleichgewichts und übergreifenden Konsenses stammt von Rawls, wird von ihm aber unter stark idealisierten Bedingungen angewendet. Vgl. etwa Rawls (2006, SS 59, 60). Für eine stärker pragmatische Lesart plädiert Sen (2010: 153-157). Rawls selbst hat seine Gerechtigkeitstheorie in späteren Werken als politische Theorie verstanden, was jedoch kontrovers diskutiert wird. Vgl. dazu etwa Nussbaum (2010). Seine Methode des Überlegungsgleichgewichts wird jedenfalls insbesondere im Kontext der angewandten Ethik auch auf moralische Fragen angewendet. Vgl. zur Diskussion Düwell (2008: 91-99) sowie Tetens (2011).

Diese Form der Reflexion lässt sich mit Scanlon (1998: 195-197) als der Versuch beschreiben, eine Lösung zu finden, die niemand mehr vernünftigerweise zurückweisen kann. Sen (2010: 72_ 79) konzeptionalisiert diese Reflexion in Anlehnung an Adam Smith als die Orientierung am Standpunkt eines unbeteiligten Beobachters.

10 Dieser minimalethische Ansatz kann daher als eine praktisch orientierte Diskursethik ohne Letztbegründungsanspruch verstanden werden, wie sie der frühe Habermas (1981 Bd. 2: 182ff., 576ff.) vertreten hat und im Anschluss daran beispielsweise Seyla Benhabib (2000) und Iris Young (2000). 
Idee der Menschenwürde und Menschenrechte im 20. Jahrhundert genauso verstehen. Inzwischen stimmen viele Menschen diesen moralischen Grundsätzen aus einer reflektierten Haltung heraus zu. ${ }^{11}$ Daher eignen sie sich auch gut als moralischer Maßstab für eine Konsumenten- und Unternehmensverantwortung (vgl. Neuhäuser/Hübscher 2010).

Gerade die Elemente der vernünftigen Reflexion und Konsensorientierung machen die moralische Verantwortung so wichtig und fügen der politischen und rechtlichen Verantwortung etwas Wesentliches hinzu. Dies gilt auch für Konsumenten und Unternehmen. Später wird sich noch zeigen, dass diese Dimension der moralischen Verantwortung die wechselseitige Abhängigkeit von Konsumenten- und Unternehmensverantwortung besonders deutlich zum Vorschein bringt. Zuvor möchte ich jedoch noch die rechtliche und politische Verantwortung von Konsumenten und Unternehmen in ihrer klassischen Gestalt, also ohne unmittelbaren Bezug zur moralischen Verantwortung, darstellen und zeigen, warum sie ohne Moral defizitär bleiben.

\section{Die rechtliche und politische Verantwortung von Konsumenten und Un- ternehmen}

Aus rechtlicher Perspektive sind Konsumenten und Unternehmen ausschließlich dafür verantwortlich, sich an das geltende Recht zu halten. Eine besondere Konsumentenverantwortung ergibt sich daraus nicht, außer in einem negativen Sinne, dass Konsumenten für ihren Konsum über die rechtlichen Regelungen hinaus selbst Sorge tragen müssen. Ob sie beispielsweise gesund, fair oder biologisch konsumieren, ist dann vollständig ihrer eigenen Verantwortung, aber auch ihrer Entscheidungshoheit überlassen. Dies macht den rechtlichen Kern der Konsumentensouveränität auf freien Märkten aus, denn in die freien Kaufentscheidungen von Konsumenten wird jenseits der rechtlichen Beschränkung von Unternehmen und erlaubten Produkten nicht eingegriffen (vgl. Heath 2005; Booms 2008).

Unternehmen werden zwar viel stärker reguliert und sie müssen sich an alle möglichen Normen, Standards und Beschränkungen halten. Aber letztlich bedeutet dies ebenfalls nur, dass Unternehmen den geltenden Gesetzen unterworfen sind und darüber hinaus in ihren Marktaktivitäten vollkommen frei bleiben (vgl. Neuhäuser 2011a: 90ff.). Ob

So stellen Menke und Pollmann (2007: 42-49) die praktische Durchsetzung der Menschenrechte nach dem Zweiten Weltkrieg dar. Natürlich gibt es trotz der breiten Zustimmung in Bezug auf die Menschenrechte auch grundsätzliche philosophische Streitfragen. Derzeit werden insbesondere vier Fragen kontrovers diskutiert: 1. Sind die Menschenrechte nur institutionell oder auch interaktional zu verstehen? 2. Verpflichten die Menschenrechte nur Staaten oder auch andere Akteure? 3. Gelten die Menschenrechte unmittelbar politisch oder haben sie eine moralische Grundlage? 4. Gibt es nur negative oder auch positive Pflichten, die mit den Menschenrechten korrespondieren? Vgl. dazu insbesondere Pogge (2002), Beitz (2009), Beitz/Goodin (2009), Griffin (2009). Ich selbst vertrete eine maximalistische Konzeption, wonach die Menschenrechte auch interaktional zu verstehen sind, auch nicht-staatliche Akteure verpflichten, eine moralische Grundlage besitzen und positive Pflichten generieren. Das kann ich hier nicht verteidigen, ist allerdings für die vorliegende Argumentation auch nicht zentral, weil es nur um den strukturellen Zusammenhang von Konsumenten- und Unternehmensverantwortung geht und nicht um die konkreten Pflichten. Vgl. zu meiner Position Neuhäuser (2011: 188-227). 
sie sich am Markt engagieren, um innerhalb der geltenden Gesetze ihren Profit zu maximieren oder ob sie mit ihren wirtschaftlichen Aktivitäten auch soziale Ziele verfolgen, bleibt ganz ihnen überlassen. Gerade wegen dieser bloß negativen Pflicht, dem Gesetz zu folgen, und der ansonsten vollkommenen Freiheit von Konsumenten und Unternehmen besteht aus rechtlicher Perspektive kein besonderer Zusammenhang zwischen der Verantwortung von Konsumenten und der Verantwortung von Unternehmen.

Natürlich können Konsumenten und Unternehmen darauf drängen, Gesetze zu ändern. Über ihre Lobbyarbeit tun sie das auch regelmäßig und aufgrund asymmetrischer Machtverhältnisse mit unterschiedlichem Erfolg. Doch das hat mit rechtlicher Verantwortung bereits nichts mehr zu tun, sondern ist Ausdruck ihrer politischen oder moralischen Verantwortung bzw. Verantwortungslosigkeit. Um eine rein politische Verantwortung handelt es sich dann, wenn sich ihr Verhalten an legitimen politischen Normen und Verfahren orientiert. Es ist nicht ganz leicht zu bestimmen, was als politisch legitim gelten kann und was nicht. Ein möglicher Maßstab wäre dabei die geltende Praxis. Für die Mehrzahl der Unternehmen besteht die in klassischer Interessenpolitik, also dem Einfluss auf politische Entscheidungsprozesse, um den eigenen unmittelbaren ökonomischen Interessen möglichst viele Vorteile zu verschaffen (vgl. Dahl 1985: 55; Dahl 1989: 311-321).

Die Mehrzahl der Konsumenten hingegen verzichtet mehr oder weniger freiwillig auf eine aktive Interessenpolitik und kommt sozusagen ihrer politischen Eigenverantwortung kaum nach. Zwar gibt es Verbraucherverbände, die beispielsweise auf mehr Informationsrechte drängen. Im Vergleich zu den Unternehmensverbänden sind sie jedoch schlecht ausgestattet und haben nur relativ wenig freiwillig aktive Mitglieder. ${ }^{12}$ Unabhängig davon können sowohl Unternehmen als auch Konsumenten ökologische oder soziale Interessen besitzen und danach streben, diese auch politisch durchzusetzen. Allerdings ist politische Verantwortung nicht an ethische Ziele gebunden. Es kann ebenfalls Ausdruck der politischen Wahrnehmung von Verantwortung sein, im politischen Raum so weit wie möglich das bloße Eigeninteresse durchzusetzen. Genauso funktionieren zahlreiche Interessenverbände, die politische Verantwortung für ihre Interessengruppen übernehmen (vgl. von Alemann/Eckert 2006).

Dieses Ergebnis erscheint vielleicht überraschend, denn es ist durchaus auch ein normativ stärker aufgeladener Begriff von politischer Verantwortung denkbar. Demnach

Die Schwäche der Konsumentenverbände gegenüber den Unternehmensverbänden lässt sich deutlich an den unterschiedlichen Mitgliederzahlen ablesen, die in keinem Verhältnis zur der repräsentierten Gruppe stehen. So verfügt der in Brüssel als Vertreter der Verbraucherinteressen eingetragene Deutsche Konsumentenbund e.V. über etwa 2.500 Mitglieder (http://www.konsumentenbund.de/anbieterkennzeichnung, zuletzt abgerufen am 29.06.2012.). Der Bundesverband mittelständische Wirtschaft - Unternehmerverband Deutschland e.V. (BVMW) hingegen verfügt über mehr als 150.000 Mitglieder (http://www.bvmw.de/der-bvmw.html, zuletzt abgerufen am 26.09.2012) und der Handelsverband Deutschland e.V. (HDE) verfügt über mehr als 100.000 Mitglieder (http://www.einzelhandel.de/pb/site/hde/node/10001/Lde/aufgaben.html, zuletzt abgerufen am 26.09.2012). 
wären nicht alle, sondern nur bestimmte normativ ausgezeichnete Interessen legitim. ${ }^{13}$ Die Interessen der hegemonialen Wirtschaftslobby beispielsweise wären es nicht, die Interessen kritischer und progressiver Konsumenten hingegen schon. Allerdings ist unklar, woher der Bewertungsmaßstab für diese Differenzierung kommen soll. Solange politische Legitimität nur deskriptiv verstanden wird, muss auch zugestanden werden, dass die ausschließliche Vertretung von profitorientierten Unternehmensinteressen legitimer Teil des gegenwärtigen politischen Spiels ist (vgl. Held 2006: 125-184). Wer das zurückweisen möchte, braucht dafür einen externen normativen Maßstab und den liefert nur eine moralische Bewertung politischer Legitimität.

Dann jedoch ist gar nicht mehr nur von politischer Verantwortung die Rede, sondern von moralischer Verantwortung im politischen Raum. Spätestens hier also zeigt sich die zentrale Bedeutung der moralischen Dimension von Verantwortung, wenn es um fairen und ökologischen Konsum geht. Es zeigt sich dabei deutlich, wie die verschiedenen Dimensionen der Verantwortung praktisch miteinander verstrickt sein können: Bestimmte Gruppen können aus moralischen Gründen auf politische Prozesse Einfluss nehmen, um die rechtliche Regulierung und damit die wirtschaftliche Rahmenordnung zu ändern. So können Konsumenten ein moralisches Recht auf mehr Information und Transparenz behaupten und dies etwa über Medien in den politischen Prozess einspeisen, um eine Änderung der Rahmenordnung zu bewirken. Es ist für solche Prozesse wichtig, die verschiedenen Dimensionen der Verantwortung analytisch auseinanderzuhalten, weil nur so ihr praktisches Zusammenspiel sichtbar wird. Immerhin ändert sich die Rahmenordnung nicht von alleine und Aufgabe einer kritischen Perspektive der angewandten Ethik ist es, das Potential für solche Reformen offenzulegen (vgl. Neuhäuser 2012).

Entsprechend gibt es auch bei der rein politischen Verantwortung keinen besonderen Zusammenhang zwischen der Verantwortung von Unternehmen und Konsumenten. Zwar können Unternehmen durch Werbung und Konsumenten durch ihre Kaufentscheidungen Einfluss auf die Interessen der jeweils anderen Gruppe nehmen, aber dies kommuniziert zumindest offiziell keinen Anspruch darauf, bestehende Interessen zu ändern. Stattdessen bleiben diese Interessen unangetastet und sollen nur durch äußeren Einfluss in gewünschte Richtungen gelenkt werden. Die politische Verantwortung wird also nicht auf vernünftige Weise wie bei der Moral in ein Überlegungsgleichgewicht gebracht, sondern Interessen werden nur durch Sanktionsmechanismen äußerlich beeinflusst.

Die Diskussion der bloß rechtlichen und politischen Verantwortung von Unternehmen und Konsumenten hat zwei eher ernüchternde Ergebnisse gebracht. Erstens gibt es weder eine besondere rechtliche noch eine besondere politische Verantwortung dafür, fairen und ökologischen Konsum zu befördern. Vielmehr besteht nur die rechtliche Verantwortung, sich an die Gesetze zu halten und eine politische Eigenverantwortung, sich für die eigenen Interessen einzusetzen. Zweitens gibt es in beiden Hin-

13 Diese Position wird etwa von Hannah Arendt (1987) vertreten, damit vertritt sie jedoch einen moralisch aufgeladenen Begriff von Politik, wie Iris Marion Young (2011: 86-93) richtig herausgestellt hat. 
sichten keinen besonderen Zusammenhang zwischen der Verantwortung von Konsumenten und Unternehmen.

\section{Die moralische Verantwortung von Konsumenten und Unternehmen}

Die zentrale Stellung der moralischen Verantwortung von Konsumenten und Unternehmen, auch für die Bereiche Politik und Recht und ihre vernünftige Transformation, hat sich im vorherigen Abschnitt bereits angedeutet. Zunächst besteht für Konsumenten erst einmal eine unmittelbar moralische Verantwortung, ökologisch und fair zu konsumieren. Für Unternehmen hingegen besteht eine unmittelbar moralische Verantwortung, ökologisch und fair zu produzieren und zu handeln. Wenn sie dies nicht tun, dann ist es angebracht, ihnen moralische Vorwürfe zu machen. ${ }^{14}$ Allerdings können sich Konsumenten und Unternehmen auch rechtfertigen oder zumindest entschuldigen, wenn sie diesen Ansprüchen nicht gerecht werden. So werden Konsumenten beispielsweise durch ihre Budgetbeschränkungen klare Grenzen gesetzt, da faire und ökologische Produkte deutlich teurer sind als andere. Wer weniger verdient, hat dann auch eine schwächere Verantwortung, fair und ökologisch einzukaufen. Unternehmen können in bestimmten Fällen auf Konkurrenzdruck und andere Marktzwänge verweisen, die ihre Verantwortung einschränken. Dies sind nur zwei Beispiele für eine Vielzahl von Beschränkungen und Zwängen, die von Verantwortung entlasten. ${ }^{15}$

Trotzdem bleibt für Unternehmen und Konsumenten stets ein individuell verschieden großer Handlungsspielraum bestehen, um ihrer moralischen Verantwortung nachzukommen. Innerhalb dieses Handlungsspielraums sind sie dazu verpflichtet, moralisch richtig zu handeln und es bleibt keine Entschuldigung durch den Verweis auf äußere Zwänge mehr (vgl. Stoecker 2007). Zu diesem Spielraum gehört es in vielen Fällen auch, aus moralischer Perspektive auf die politische Legitimität von Interessen und die Gesetzgebung Einfluss zu nehmen. Es besteht dann eine moralische Verantwortung von Konsumenten und Unternehmen, die Rahmenbedingungen für ein ökologisches und soziales Marktgeschehen nach Möglichkeit zu verbessern. Beide Gruppen können aus moralischer Perspektive auf bessere Gesetze drängen und bestimmte Interessen, beispielsweise der rücksichtslosen Profitmaximierung, im politischen Diskurs als illegitim ausweisen.

Dieser Punkt ist wichtig und wird insbesondere von Iris Marion Young stark betont. Moralische Verantwortung bezieht sich nicht nur auf eigenes Handeln, sondern auch auf strukturelle Hintergrundbedingungen. Young spricht dann von einer politischen Verantwortung für kollektives Handeln. Dem lässt sich entgegenhalten, dass es tatsächlich um eine moralische Verantwortung für kollektives politisches Handeln geht,

14 Hier zeigt sich die Nähe des moralischen Verantwortungsbegriffs zum Pflichtenbegriff: Man ist dazu verpflichtet, seiner moralischen Verantwortung nachzukommen, es ist nicht beliebig und nicht „supererogatorisch“, also über das, was verlangt werden kann, hinausgehend. Vgl. dazu klassisch: Feinberg (1980).

15 Zu Entschuldigungen und Rechtfertigungen bei Unternehmen: Neuhäuser (2011a: 212-220) und zu der Vielzahl der zu berücksichtigenden individuellen Faktoren bei Konsumenten: Fischer und Sommer (2011). 
weil diese Verantwortung auch bei Young an moralischen Maßstäben bemessen wird. ${ }^{16}$ Auf diese Weise können sich Konsumenten in ihrem Handeln als Konsumenten, aber auch als Bürger beispielsweise über Konsumentenverbände darauf verständigen, die Rahmenordnung so zu ändern, dass ein moralisch motivierter Konsum leichter wird, weil mehr Informationen über Unternehmen zur Verfügung stehen und leichter zugänglich sind.

Eine rechtliche und politische Verantwortung für faires und ökologisches Wirtschaften besteht tatsächlich also nur als eine, auf Recht und Politik bezogene, moralische Verantwortung. Zugleich ist es von ganz entscheidender Bedeutung, diese erweiterte Dimension der moralischen Verantwortung von Konsumenten und Unternehmen zu berücksichtigen. Dafür gibt es zwei gewichtige Gründe. Der erste Grund hat mit dem schwerwiegenden Vorwurf der relativen Wirkungslosigkeit einer bloß moralischen Verantwortung von Unternehmen und Konsumenten zu tun. Der zweite Grund besteht darin, dass nur durch eine Verschränkung der moralischen mit der politischen und rechtlichen Verantwortung auch die Verschränkung von Konsumenten- und Unternehmensverantwortung ihr volles Potential entfalten kann. Dem ersten Punkt wende ich mich in diesem und dem zweiten im nächsten Abschnitt zu.

Gegen eine moralische Verantwortung, insbesondere von Konsumenten, aber auch von Unternehmen, wird häufig eingewendet, dass sie relativ wirkungslos und bloß eine „Fahrradbremse am Interkontinentalflugzeug“ sei. ${ }^{17}$ Der Grund für diese ziemlich skeptische Vermutung besteht darin, dass individuelles Handeln von Konsumenten und selbst von größeren Unternehmen wirkungslos verpufft. Sehr gut lässt sich das am Beispiel des nachhaltigen Konsums, aber auch der nachhaltigen Produktion zeigen. Für den Klimawandel ist es völlig gleichgültig, was für ein Auto sich jemand kauft und wie viel er damit fährt. Seine individuelle $\mathrm{CO}_{2}$-Produktion hat absolut keine Auswirkungen auf Intensität und Folgen des Klimawandels. Dasselbe gilt sogar für große Automobilunternehmen. Sogar wenn sich ein großer Konzern dafür entscheiden würde, seine Produktion aus Gründen der Nachhaltigkeit sofort einzustellen, dann hätte dies keinen unmittelbaren Effekt auf den Klimawandel (vgl. Neuhäuser 2011a: 238ff.). Selbst wenn wir also eine menschenrechtsbasierte Verantwortung gegenüber zukünftigen Generationen zugestehen, folgt daraus für individuelle Autokäufer und -hersteller keine unmittelbare Verantwortung, weil ihr Handeln keinen direkten Effekt auf diese

16 Young (2011) folgt in diesem Punkt Hannah Arendt (1986), die jedes auf Politik bezogene Handeln als politisch bezeichnet. Das ist jedoch eine der Antike entnommene Weise, über Politik als Teil der Tugendethik zu sprechen. Bloße Interessenpolitik ist in diesem Konzept schon rein begrifflich nicht möglich. Daher trenne ich stärker zwischen Moral und Politik, um den Begriff des Politischen von stärker moralischen Ansprüchen auf rein begrifflicher Ebene frei zu halten.

17 Diese Formulierung hat Ulrich Beck immer wieder in Anschlag gebracht (vgl. z.B. 2007: 73). Statt moralischer Verantwortung und Ethik fordert er den Aufbau einer politischen Gegenmacht und Konsumenten sollen sich lieber als politische Akteure begreifen (vgl. Beck 2011: 349). Als ziemlich wirkungslos kritisiert auch Grunwald (2010) die Konsumethik und plädiert stattdessen für strukturelle Reformen. Beide unterschätzen jedoch die Bedeutung der Moral als Ressource in politischen Konflikten und als Impuls für Strukturreformen. Für eine Replik auf Grunwald: Bilharz/Fricke/Schrader (2011). 
zukünftigen Generationen haben kann. ${ }^{18}$ Stattdessen bedürfe es eines grundlegenden Wandels des Wirtschaftssystems hin zu mehr Nachhaltigkeit und Gerechtigkeit, so argumentieren die Kritiker der Konsumenten- und Unternehmensverantwortung (vgl. Rosa 2011; Grunwald 2010). Einerseits stimmt es, dass sich das Wirtschaftssystem und seine Rahmenordnung grundsätzlich ändern müssen, wenn es tatsächlich nachhaltig und fair sein soll. Doch dies steht nicht in Opposition zur Konsumenten- und Unternehmensverantwortung, kann es vielleicht auch gar nicht. Tatsächlich sind es zwei gewichtige Gründe, die sich gegen diesen Vorwurf der Wirkungslosigkeit anführen lassen.

Erstens gibt es viele Bereiche, in denen die Übernahme von Verantwortung durch Konsumenten und Unternehmen alles andere als wirkungslos ist. Dies ist offensichtlich bei multinationalen Konzernen der Fall, die schädigendes Verhalten unterlassen können und oft weitreichende Hilfsmöglichkeiten haben; etwa Pharmakonzerne bei der Verteilung von Medikamenten an absolut arme Menschen (vgl. Pogge 2008). Aber auch Konsumenten können direkte Effekte erzielen. Wenn sie fair gehandelte und tierfreundlich produzierte Waren erwerben, dann hat dies sichtbare Auswirkungen. Einige Menschen verdienen mehr Geld und können selbstbestimmter leben und einige Tiere werden artgerechter gehalten. Diese Effekte produzieren keinen Systemwechsel, aber sie ersparen wirklichen Menschen und Tieren viel Leid und sind daher aus moralischer Perspektive durchaus wirkungsvoll und auch geboten.

Die zweite Replik auf den Vorwurf der Wirkungslosigkeit einer moralischen Konsumenten- und Unternehmensverantwortung verweist unmittelbar auf die politische und rechtliche Dimension moralischer Verantwortung, also der moralischen Verantwortung für die Änderung der Hintergrundbedingungen (vgl. Lichtenberg 2009). Wenn Unternehmen und Konsumenten ihrer moralischen Verantwortung nachkommen, dann hat dies immer auch eine politische und potentiell rechtliche Wirkung. Durch die symbolische Kraft ihres an moralischer Verantwortung orientierten wirtschaftlichen Handelns können sie unmittelbar Einfluss darauf nehmen, was als politisch legitim gilt und was nicht. Wenn beispielsweise immer mehr Menschen fair gehandelten Kaffee kaufen, dann erscheint es auch immer weniger legitim, genau dies nicht zu tun. Diese für sich genommen noch recht schwache politische Symbolik lässt sich durch eine entsprechende politische Kommunikation verstärken. Es wird dann politisch gefordert, was moralisch richtig ist, in diesem Fall also fair gehandelten Kaffee zu kaufen (vgl. Young 2006; Beck 2010). Die rechtliche Dimension kommt zusätzlich noch ins Spiel, weil sich nicht nur moralisches Handeln in der Wirtschaft politisch fordern lässt, sondern auch eine gesetzliche Regulierung. Konsumenten und Unternehmen können deutlich machen, dass ihnen eine moralische und selbst durch politischen Druck gestützte, aber letztlich doch nur schwach sanktionierte Verantwortung, nicht ausreicht und sie stattdessen auf eine rechtliche Regelung drängen. Dies liegt natürlich immer dann nahe, wenn eine Tragik der Allmende besonders gravierend wäre und kollektives Handeln unterminierendes Trittbrettfahren besonders wahrscheinlich ist (vgl. Ostrom

18 Daraus ergibt sich, dass die Konsumenten keine entsprechenden Pflichten haben können. Denn ein allgemein anerkannter philosophischer Grundsatz lautet: Das moralische Sollen impliziert das faktische Können (vgl. Copp 2003). 
2011). Freilich ersetzt diese Forderung nach rechtlicher Regulierung die individuelle moralische Verantwortung nicht vollständig, sondern soll sie vielmehr institutionell stützen. Dadurch wird zugleich auch zum Ausdruck gebracht, dass es eben nicht nur um Interessenpolitik zur Durchsetzung einer Gesetzgebung, die nur für einen selbst vorteilhaft ist, sondern um moralisch fundierte Gesetze geht.

Dieser Punkt lässt sich an dem bereits mehrmals erwähnten Beispiel der Informationspflicht verdeutlichen. Derzeit sind für Konsumenten die Produktionsprozesse und Arbeitsbedingungen von Unternehmen oft sehr intransparent. Das macht es schwierig, moralisch motivierte Kaufentscheidungen zu treffen oder öffentlich Kritik zu üben. Vor diesem Hintergrund liegt es für Konsumenten und ihre Verbände, gemeinsam mit moralisch engagierten Unternehmen, nahe, sich für bessere Informationsrechte, also eine veränderte Rahmenordnung einzusetzen. Wenn sie dies öffentlichkeitswirksam im politischen Raum tun, beispielsweise indem sie Unternehmen zu mehr Transparenz auffordern, dann haben sie eine realistische Chance, dass Politiker dieses Anliegen aufgreifen und in den Gesetzgebungsprozess einspeisen. Das moralische Anliegen der Konsumenten kann also eine politische und rechtliche Wirkung entfalten und es lässt sich durchaus behaupten, dass eine moralische Verantwortung für diese politische Veränderung der Hintergrundstruktur besteht. ${ }^{19}$

Allerdings können die Kritiker einer Konsumenten- und Unternehmensverantwortung an dieser Stelle wiederum zwei grundsätzliche Dinge einwenden. Erstens besteht noch immer das Problem, dass es für moralische Maßstäbe keine allgemeinverbindlichen Begründungen zu geben scheint, insbesondere wenn nicht unmittelbar Menschenrechtsfragen betroffen sind. Zweitens scheinen die einzelnen verantwortungsbereiten Akteure und insbesondere ethisch orientierte Konsumenten viel zu machtlos, um den Diskurs der politischen Legitimität oder sogar die Gesetzgebung wirkungsvoll beeinflussen zu können. Die Antwort auf diesen zweiten Einwand stützt sich wieder auf die Verschränkung von Konsumenten- und Unternehmensverantwortung und erfolgt daher erst im nächsten Abschnitt. Die Antwort auf den ersten Einwand hingegen trifft in zentraler Weise die Bedeutung moralischer Verantwortung, stellt er ihre normative Verbindlichkeit doch grundsätzlich in Frage.

Eine vollständige Antwort auf diesen ersten Einwand würde eine grundsätzliche Auseinandersetzung mit der Rolle von Moral in pluralistischen spätmodernen Gesellschaften erfordern und lässt sich hier nicht leisten. Dennoch sollen einige Missverständnisse aus dem Weg geräumt werden, um zumindest plausibel zu machen, warum Moral keine wirkungslose Angelegenheit ist, sondern eine zentrale Ressource zur Modifikation des Wirtschaftssystems sein kann (vgl. Neuhäuser 2011a: 221). Zunächst gilt es noch einmal festzuhalten, dass eine Letztbegründung moralischer Werte angesichts eines vernünftigen Pluralismus an Werten nicht möglich ist. Allerdings ist solch ein Begründungsanspruch auch gar nicht nötig. Um moralische Gründe für wirtschaftli-

19 Ein möglicher Einwand an dieser Stelle wäre, dass hier die Rolle des Konsumenten mit der Rolle des Bürgers verwechselt wird. Im nächsten Abschnitt wird jedoch deutlich werden, dass Bürger ihrer Bürgerverantwortung auch in der Rolle des Konsumenten nachkommen können. Konsumentenverantwortung ist also Teil der Bürgerverantwortung. 
ches Handeln von Konsumenten und Unternehmen zu identifizieren, bedarf es vielmehr nur reflexiver Kompetenzen und der Orientierung an einem Überlegungsgleichgewicht in einem praktischen moralischen Diskurs (vgl. Birnbacher 2003: 92-98).

Konkret bedeutet dies, dass Konsumenten und Unternehmen erstens ihre Handlungsgründe reflektieren und zweitens dem kritischen Urteil anderer Akteure aussetzen müssen. Nur wenn ihre Handlungsgründe einer öffentlichen Überprüfung standhalten, dann sind sie moralisch akzeptabel und fließen in ein Überlegungsgleichgewicht ein. Die zusätzliche Funktion dieses Überlegungsgleichgewichts besteht darin, einen relativ kohärenten moralischen Maßstab herzustellen, indem verschiedene moralische Gründe in einen Zusammenhang gesetzt werden. Dies alles geschieht in einem öffentlichen Raum; Moral ist daher keine Privatsache, sondern eine öffentliche Ressource. So werden beispielsweise moralische Themen in den Medien aufgegriffen und die Frage, welche Form von Konsum erlaubt oder geboten ist, wird immer wieder, auch unter Beteiligung von Moralphilosophen, öffentlich diskutiert und stößt auf reges Interesse. ${ }^{20}$

Auf diese Weise gelangen Akteure zu moralischen Normen, die sich deutlich von unreflektierten Werthaltungen unterscheiden. Übrigens bedeutet das nicht, dass diese Akteure immer aus moralischen Gründen handeln müssen. Oft reicht es moralkonform, aber aus anderen Gründen zu handeln. Manchmal überwiegen natürlich auch andere, moralfeindliche Handlungsgründe. Dann handeln die Akteure tatsächlich nicht moralisch, doch das bedeutet natürlich auch nicht, dass sie keine moralischen Maßstäbe haben können, die sie faktisch einfach missachten.

Moralisches Handeln ist also nicht einfach jedes wertbezogene Handeln, wie Moralsoziologen es manchmal behaupten. ${ }^{21}$ Vielmehr ist moralisches Handeln, auch von Unternehmen und Konsumenten, immer an einen öffentlichen Begründungsdiskurs gebunden (vgl. Young 2011: 95ff.). Darauf verweist auch der dialogische Charakter von Verantwortung, denn Akteure schreiben sich Verantwortung wechselseitig zu. Genau hier wird auch deutlich, warum Konsumenten- und Unternehmensverantwortung eng miteinander verschränkt sind und nur gemeinsam in einem öffentlichen Raum ihre Wirkung entfalten können.

\section{Zum Verhältnis von Konsumenten- und Unternehmensverantwortung}

Zumindest andeutungsweise hat sich bereits gezeigt, dass eine Konsumentenverantwortung ihr volles Potential nur dann entfalten kann, wenn sie im Zusammenhang mit einer Unternehmensverantwortung gesehen wird. Überraschender ist vielleicht das Ergebnis, dass umgekehrt auch eine Unternehmensverantwortung auf eine Konsumentenverantwortung angewiesen ist. Doch warum ist das eigentlich so? Und was

20 Dafür spricht nicht zuletzt, dass laut Jahresbericht 2011/12 von TransFair der Fair-Trade-Markt in Deutschland um 18 Prozent gewachsen ist. Inzwischen gibt es bereits Fernsehwerbung in der für den Kauf von Produkten mit dem Fairtrade-Siegel geworben wird.

21 So stellen jedes wertbezogene Handeln von Konsumenten als moralisches Handeln beispielsweise Stehr (2007: 69ff.), Adolf und Stehr (2011) sowie Hellmann $(2008,2011)$ dar. Doch selbst aus der subjektiven Perspektive der Konsumenten gibt es einen Unterschied zwischen bloß wertbezogenem und moralisch gerechtfertigtem Handeln. Vgl. dazu Celikates (2009: 116ff.). 
genau ist damit gemeint, dass die Verantwortung dieser beiden Akteursgruppen miteinander verschränkt ist? Eine gewisse Verknüpfung liegt vielleicht schon deshalb nahe, weil es einen grundlegenden ökonomischen Zusammenhang zwischen Angebot und Nachfrage gibt. Tatsächlich kann die dem Wirtschaftssystem eingeschriebene Logik vom Kaufen und Verkaufen bzw. Zahlen und Nichtzahlen nur dann moralisch aufgeladen werden, wenn sich die Akteure wechselseitig als verantwortliche Akteure adressieren. ${ }^{22}$

Konsumentenverantwortung beinhaltet daher in einem ganz entscheidenden Maße auch, Unternehmen als verantwortliche Akteure zu adressieren. Wenn Konsumenten sich einfach nur dafür entscheiden, fair gehandelte und nachhaltig produzierte Waren zu kaufen, dann handelt es sich dabei aus Sicht der Unternehmen zunächst einfach nur um ganz beliebige subjektive Präferenzen. Solange die einzelne Kaufhandlung von Konsumenten isoliert bleibt, verpufft sie tatsächlich relativ wirkungslos im politischen Raum. ${ }^{23}$ In diesem Fall verwundert es auch nicht, dass bei Konsumenten eine deutliche Lücke zwischen ihren eigenen moralischen Ansprüchen und ihrem tatsächlichen Verhalten klafft (vgl. Borgstedt/Reusswig 2010; Fischer/Sommer 2011). Was soll die Mühe, wenn das individuelle Verhalten derart irrelevant ist? Der Schluss, dass man dann einfach anderen Kaufimpulsen nachgeben könne, liegt angesichts dieser Hilflosigkeit ziemlich nahe. Dies gilt besonders dann, wenn es Konsumenten wirklich um effektive Verantwortung und nicht einfach nur um ihre gute Gesinnung geht. ${ }^{24}$

Anders erscheint jedoch die Lage, wenn Konsumenten Unternehmen unmittelbar als moralische Akteure adressieren. Dadurch werden Prozesse der wechselseitigen Beund Verstärkung in der Übernahme von Verantwortung möglich. Unternehmen werden in einen öffentlichen Diskurs einbezogen, in dem es auch um die moralischen Grundlagen der politischen Legitimität und rechtlichen Ordnung geht. ${ }^{25}$ Einzelne Kaufakte sind dann nicht mehr isoliert zu betrachten, sondern Teil einer politischen Strategie. Die Rolle von Konsumenten verschmilzt ein Stück weit mit der Rolle von Bürgern: Als Konsumenten handeln Bürger durch ihre Kaufakte politisch. Man könnte daher auch sagen, dass Konsumentenverantwortung dann Teil der Bürgerverantwortung ist. Allerdings ist zu konstatieren, dass sich diese Entwicklung einer Politisierung des moralischen Konsums erst am Anfang befindet (vgl. Barber 2007: 289f.). Wenn verantwortlicher Konsum jedoch mehr leisten soll, als vereinzelte Effekte auf

22 Anders ausgedrückt muss man die systemtheoretische Perspektive auf Wirtschaft, wie sie insbesondere Luhmann (1994: 43-90) entwickelt hat, durch eine akteursbezogene Perspektive durchbrechen, weil nur so Moral und Verantwortung überhaupt relevante Maßstäbe sein können. Aus diesem Grund können die Konsumenten auch nicht von vornherein als Gruppe konzeptionalisiert werden, die selbst verantwortungsfähig ist. Zunächst handeln Konsumenten nur als Aggregate, also unkoordiniert und sind daher nicht kollektiv verantwortungsfähig. Erst wenn sie sich politisch koordinieren, erfüllen sie überhaupt die handlungstheoretischen Voraussetzungen für Verantwortungsfähigkeit. Vgl. dazu French (1984).

24 Konsumenten müssen also eine Verantwortungsethik und nicht eine Gesinnungsethik im Sinne von Max Weber (1988) verfolgen.

25 Solch eine Perspektive auf den öffentlichen Diskurs, in dem Moral und Gerechtigkeit selbst Ressourcen des Konflikts sind, entwickelte Iris Young (2000: 157-179). 
ökologische Produktion und die Lebensqualität einiger Produzenten zu bewirken, dann bedarf es genau dieser Politisierung der Konsumethik.

Damit diese Politisierung eines moralisch verantwortlichen Konsums gelingen kann, ist allerdings einer Koordination der Aktivitäten individueller Konsumenten nötig. Vereinzelte Kaufakte ohne Zusammenhang können dafür zwar einen Ausgangspunkt bilden, reichen letztlich aber nicht aus. Zur Verantwortung von Konsumenten gehört es daher gleichzeitig auch, sich zu organisieren, beispielsweise in entsprechenden NGO und Bürgerbewegungen. Dabei wird es naturgemäß einige Vorreiter geben, die erforderliche Grundlagen schaffen, und daneben viele eher passiv unterstützende Mitstreiter. Dennoch ist dieser Schritt für eine politische Konsumethik wesentlich. Denn nur durch ihn wird eine Strategie möglich, die Unternehmen direkt als moralisch verantwortliche Akteure adressiert und von ihnen verlangt, sich allmählich auf einen fairen und nachhaltigen Wandel in Produktion und Handel einzustellen bzw. dies sogar mit voranzutreiben. ${ }^{26}$

Tatsächlich wäre es die falsche Perspektive, Unternehmen nur als passive Rezipienten von moralischen Ansprüchen zu sehen. Sie selbst können erstens aus eigenem Antrieb Verantwortung übernehmen und zweitens sogar selbst anderen Marktakteuren Verantwortung zuschreiben, seien es andere Unternehmen oder Konsumenten. Dies gilt insbesondere für Unternehmen, die schon fair und nachhaltig wirtschaften. Sie können andere Unternehmen ermuntern, es ihnen gleich zu tun; dies gilt beispielsweise für eng zusammenarbeitende mittelständische Unternehmen. Sie können aber auch Konsumenten darauf hinweisen, dass sie im Rahmen ihrer Möglichkeiten eine Verantwortung dafür haben, fair und nachhaltig zu konsumieren. Durch die wechselseitige Zuweisung von Verantwortung kann es Konsumenten und Unternehmen gelingen - so die konsumethische Hoffnung - mehr Marktanteile für eine faire und nachhaltige Wirtschaft zu gewinnen.

Dies wird eine rechtliche Regelung nicht ersetzen, denn manche Dinge lassen sich nur durch Gesetze steuern; dies gilt insbesondere in sehr komplexen und kostenintensiven Zusammenhängen. Doch die Übernahme von Verantwortung durch Konsumenten und Unternehmen schließt zugleich ein, den Gesetzgeber politisch zu adressieren und die eigene Bereitschaft für entsprechende rechtliche Regelungen zu signalisieren, statt sich ihnen gegenüber passiv zu verhalten oder sogar zu widersetzen. Gerade deswegen ist die politische Dimension der moralischen Verantwortung auch so zentral. Denn in dem öffentlichen politischen Raum können sich Konsumenten und Unternehmen über einzelne Kaufakte hinaus wechselseitig als moralische Akteure adressieren und in einen durchaus kooperativ orientierten Dialog über Verantwortung eintreten. Die Bereitschaft zu solch einem Dialog lässt sich durch moralisch aufgeladene Angebote und Nachfragen am Markt symbolisch zum Ausdruck bringen.

26 Eigentlich vertritt Peter Ulrich (1997: 289ff.) in seiner integrativen Wirtschaftsethik ein Konzept des Wirtschaftsbürgers, der ebenfalls aus moralischen Gründen politisch handelt. Er unterschätzt in seinem diskursethischen Modell meiner Einschätzung nach jedoch die praktische Dimension der konflikthaften politischen Auseinandersetzung, wie beispielsweise Young (1990: 116ff.; 2000) sie betont. Etwas Ähnliches wie für Ulrich gilt meiner Einschätzung nach auch für Ulf Schrader (2007) und seine Konzeption der Konsumentenverantwortung als Bürgerverantwortung. 
Eine besondere Herausforderung für einen moralischen Diskurs über die Verantwortung von Konsumenten und Unternehmen im öffentlichen politischen Raum besteht darin, dass er nicht moralistisch in einem schlechten Sinne werden darf. Dies würde bedeuten, dass anderen Akteuren nur darum Verantwortung zugewiesen wird, um selbst gut bzw. besser dazustehen oder das eigene Gewissen zu beruhigen. Dann käme es jedoch schnell zu einer Verantwortungsdiffusion, in der nur noch irgendwelche anderen Akteure verantwortlich sind, nie jedoch man selbst und letztlich also niemand. ${ }^{27}$ Aus diesem Grund ist es wohl auch angebracht, bei der Zuweisung von Verantwortung mit Schuldvorwürfen sparsam umzugehen. Für den öffentlichen Diskurs über Verantwortung bietet sich also ein zukunftsorientierter und positiv aktivierend ausgerichteter Diskurs an, der sich normativ an den Menschenrechten orientiert. ${ }^{28}$

Solch eine Verschränkung der Verantwortung von Konsumenten und Unternehmen, freilich unter Berücksichtigung ihrer unterschiedlichen Stellung im Marktgeschehen, ist von zentraler Bedeutung für die politische Dimension der moralischen Verantwortung. Diese politische Dimension der Verantwortung ist wiederum zentral, damit es zu einer zunehmenden Koordination der Verantwortung kommt und nicht bei vereinzelten Bemühungen bleibt, die manchmal zwar eine direkte, insgesamt aber nur sehr beschränkte Wirkung entfalten können. Demgegenüber ist ein öffentlich und politisch gespanntes Netz der moralischen Verantwortung von Konsumenten und Unternehmen nötig, um eine sanfte moralische Revolution des Marktes überhaupt erst denkbar zu machen. Damit dies mittelfristig und mit zunehmender Wirkung gelingen kann, bedarf es einer Institutionalisierung, also einer politischen Verfassung der moralischen Bewegung für ein faires und ökologisches Wirtschaften. $\mathrm{Ob}$ dies geschehen wird und moralisch orientierte Konsumenten sich tatsächlich zunehmend politisieren, bleibt natürlich abzuwarten - undenkbar ist es nicht.

\section{Literaturverzeichnis}

Adolf, M./Stebr, N. (2011): Soziale Milieus und Konsum: Die Moralisierung der Märkte und ihre Kritik, in: Heidbrink, L./Schmidt, I./Ahaus, B. (Hrsg.): Die Verantwortung des Konsumenten: Über das Verhältnis von Markt, Moral und Konsum, Frankfurt a. M./New York: Campus, 245-268.

Appiah, A. (2007): Der Kosmopolit, München: C.H. Beck.

Arendt, H. (1987): Collective Responsibility, in: Bernauer, J. W. (Hrsg.): Amor Mundi: Explorations in the Faith and Thought of Hannah Arendt, Dordrecht: Martinus Nijhoff, 43-50.

Barber, B. J. (2007): Consumed: How Markets Corrupt Children, Infantilize Adults, and swallow Citizens Whole, New York/London: Norton.

$27 \quad$ Dies scheint mir die wirkliche Gefahr einer Verantwortungsdiffusion zu sein und nicht, dass alle Verantwortung miteinander teilen und deswegen niemand verantwortlich sei. Es kommt vielmehr darauf an Verantwortung fair zu verteilen.

28 Dies müssen, so argumentiert Iris Marion Young (2011: 113-122), alle emanzipativen und progressiven Bewegungen berücksichtigen, wenn sie ihre Ressentiments überwinden und ihre Bestrebungen dauerhaft implementieren wollen. Allerdings schließt das ein „blaming und shaming“ bei gravierenden Verstößen gegen die Menschenrechte nicht aus. 
Bayertz, K. (1995): Eine kurze Geschichte der Herkunft der Verantwortung, in: Bayertz, K. (Hrsg.): Verantwortung. Prinzip oder Problem?, Darmstadt: WBG.

Beck, U. (2001): Macht und Gegenmacht im globalen Zeitalter, Frankfurt a. M.: Suhrkamp.

Beck, U. (2007): Weltrisikogesellschaft: Auf der Suche nach der verlorenen Sicherheit, Frankfurt a. M.: Suhrkamp.

Beck, V. (2010): Theorizing Fairtrade from a justice-related standpoint, in: Global Justice: Theory, Practice, Rhetoric, No. 3, 1-21.

Beitz, C. (2009): The Idea of Human Rights, Oxford: Oxford University Press.

Beitz, C./ Goodin, R. (2009): Global Basic Rights, Oxford: Oxford University Press.

Benhabib, S. (2000): Kulturelle Vielfalt und demokratische Gleichheit. Politische Partizipation im Zeitalter der Globalisierung, Frankfurt a. M.: Suhrkamp.

Bilhary, M./Fircke, V./Schrader, U. (2011): Wider die Bagatellisierung der Konsumentenverantwortung, in: Gaja, Vol. 20/No. 1, 9-13.

Birnbacher, D. (2003): Analytische Einführung in die Ethik, Berlin/New York: de Gruyter.

Booms, M. (2008): Werbung und Werte - Konsumethische Überlegungen zu einem ambivalenten Verhältnis, in: Beschorner, T./Schmidt, M./Vorbohle, K./Schank, Ch. (Hrsg.): Zur Verantwortung von Unternehmen und Konsumenten, München/Mering: Hampp, 6376.

Borgstedt, S./Christ, T./Reusswig, F. (2010): Umweltbewusstsein in Deutschland: Ergebnisse einer repräsentativen Umfrage, Bundesministerium für Umwelt, Naturschutz und Reaktorsicherheit.

Busse, T. (2006): Die Einkaufsrevolution: Konsumenten entdecken ihre Macht, München: Karl Blessing.

Celikates, R. (2009): Kritik als soziale Praxis: Gesellschaftliche Selbstverständigung und kritische Theorie, Frankfurt a. M./New York: Campus.

Copp, D. (2003): Ought implies can, blameworthiness, and alternative possibilities, in: Widerker, D./McKenna, M. (Hrsg.): Moral Responsibility and Alternate Possibilities, Aldershot: Ashgate, 265-299.

Crane, A./Matten, D. (2010): Business Ethics: Managing Corporate Citizenship and Sustainability in the Age of Globalization, Oxford: Oxford University Press.

Dabl, R. (1985): A Preface to Economic Democracy, Cambridge: Polity Press.

Dabl, R. (1989): Democracy and its Critics, New Haven: Yale University Press.

Devinney, T./Auger, P./Eckhardt, G. M. (2010): The Myth of the Ethical Consumer, Cambridge: Cambridge University Press.

Düwell, M. (2008): Bioethik: Methoden, Theorien, Bereiche, Stuttgart: J.B. Metzler.

Feinberg, J. (1980): The Nature and Value of Rights, in: Feinberg, J. (Hrsg.): Rights, Justice, and the Bounds of Liberty, Princeton: Princeton University Press, 143-155.

Fischer, M./Sommer, B. (2011): Mentale und soziale Infrastrukturen - Voraussetzungen verantwortungsvollen Konsums im Kontext der Nachhaltigkeit, in: Heidbrink, L./Schmidt, I./Ahaus B. (Hrsg.): Die Verantwortung des Konsumenten: Über das Verhältnis von Markt, Moral und Konsum, Frankfurt a. M./New York: Campus, 183-202.

French, P. (1979): The Corporation as a Moral Person, in: American Philosophical Quarterly Vol. 16/No. 3, 207-215.

French, P. (1984): Collective and Corporate Responsibility, New York: Columbia University Press.

French, P. (1995): Corporate Ethics, Fort Worth: Thomson Learning.

Griffin, J. (2009): On Human Rights, Oxford: Oxford University Press. 
Grunwald, A. (2010): Wider die Privatisierung der Nachhaltigkeit: Warum ökologisch korrekter Konsum die Welt nicht retten kann, in: Gaja Vol. 19/No. 3, 178-182.

Habermas, J. (1981): Theorie des kommunikativen Handelns, Frankfurt a. M.: Suhrkamp.

Hartmann, K. (2009): Ende der Märchenstunde: Wie die Industrie die Lohas und Lifestyle-Ökos vereinnahmt, München: Karl Blessing.

Heath, J. (2005): Liberal Autonomy and Consumer Sovereignity, in: Christman, J./Anderson, J. (Hrsg.): Autonomy and the Challenges to Liberalism: New Essays, Cambridge: Cambridge University Press, 204-225.

Heath, J./Potter, A. (2005): Konsumrebellen: Der Mythos der Gegenkultur, Berlin: Der Freitag Mediengesellschaft.

Held, D. (2006): Models of Democracy, Stanford: Stanford University Press.

Hellmann, K.-U. (2008): Konsumkultur und Markenindustrie: Ein Beitrag zum Problem der Unternehmensverantwortung, in: Beschorner, T. et al. (Hrsg.): Zur Verantwortung von Unternehmen und Konsumenten, München/Mering: Hampp 2008, 77-94.

Hellmann, K.-U. (2011): Der Eigensinn der Konsumenten - Zur Frage der Verantwortung bei der Zurechnung von Konsumentenverantwortung, in: Heidbrink, L./Schmidt, I./Ahaus B. (Hrsg.): Die Verantwortung des Konsumenten: Über das Verhältnis von Markt, Moral und Konsum, Frankfurt a. M./New York: Campus, 269-284.

Heidbrink, L. (2003): Kritik der Verantwortung: Zu den Grenzen verantwortlichen Handelns in komplexen Kontexten, Weilerswist: Velbrück Wissenschaft.

Heidbrink, L./Schmidt, I./Ahaus, B. (Hrsg.) (2011): Die Verantwortung des Konsumenten: Über das Verhältnis von Markt, Moral und Konsum, Frankfurt a. M./New York: Campus.

Heidbrink, L./Schmidt, I. (2011): Das Prinzip der Konsumentenverantwortung - Grundlagen, Bedingungen und Umsetzungen verantwortlichen Konsums, in: Heidbrink, L./Schmidt, I./Ahaus, B. (Hrsg.): Die Verantwortung des Konsumenten: Über das Verhältnis von Markt, Moral und Konsum, Frankfurt a. M./New York: Campus, 25-56.

Hübscher, M. (2010): Die Firma als Nexus von Rechtfertigungskontexten: Eine normative Untersuchung zur rekursiven Simultanität von Individuen und Institutionen in der Governanceethik, Marburg: Metropolis.

Jaspers, K. (2012): Die Schuldfrage: Von der politischen Haftung Deutschlands, München: Piper.

König, W. (2008): Kleine Geschichte der Konsumgesellschaft: Konsum als Lebensform der Moderne, Stuttgart: Steiner.

Lichtenberg, J. (2009): Are There any Basic Rights?, in: Beitz, C./Goodin, R. (2009): Global Basic Rights, Oxford: Oxford University Press, 71-91.

List, C./Pettit, P. (2011): Group Agency: The Possibility, Design, and Status of Corporate Agents, Oxford: Oxford University Press.

Lohmann, G. (2002): Zur moralischen, ethischen und rechtlichen Verantwortung in Wissenschaft und Technik, in: Burckhart, H./Gronke, H. (Hrsg.): Philosophieren aus dem Diskurs. Beiträge zur Diskurspragmatik, Würzburg: Königshausen \& Neumann, 366-378.

Lubmann, N. (1994): Die Wirtschaft der Gesellschaft, Frankfurt a. M.: Suhrkamp.

Menke, C./Pollmann, A. (2007): Philosophie der Menschenrechte: Zur Einführung, Hamburg: Junius.

Neubäuser, C./Hübscher, M. (2010): Unternehmen, ihre (ethische) Governance und Menschenrechte, in: Jahrbuch für Recht und Ethik, Band 18, 349-368.

Neubäuser, C. (2011a): Verantwortung in komplexen Umwelten, in: Heimbach-Steins, M./Kruip, G. (Hrsg.): Kooperative Bildungsverantwortung. Sozialethische und pädagogische Perspektiven auf Educational Governance, Bielefeld: W. Bertelsmann, 53-66. 
Neubäuser, C. (2011b): Unternehmen als moralische Akteure, Berlin: Suhrkamp.

Neubäuser, C. (2012): Politische Ethik zwischen kritischer Theorie und angewandter Ethik, in: Jahrbuch für Recht und Ethik, Band 19, 35-50.

Nussbaum, M. C. (2010): Die Grenzen der Gerechtigkeit, München: C.H. Beck.

Ostrom, E. (2011): Handeln statt Warten: Ein mehrstufiger Ansatz zur Bewältigung des Klimaproblems, in: Leviathan, Vol. 39/No. 2, 267-278.

Peter, F. (2007): Democratic Legitimacy and Proceduralist Social Epistemology, in: Politics, Philosophy and Economics, Vol. 6/No. 3, 329-353.

Pogge, T. (2002): World Poverty and Human Rights. Cambridge/MA: Polity Press.

Pogge, T. (2008): Access to Medicines, in: public health ethics, Vol. 1/No. 2, 73-82.

Rawls, J. (2006): Gerechtigkeit als Fairness: Ein Neuentwurf, Frankfurt a. M.: Suhrkamp.

Scanlon, T. (1998): What We Owe To Each Other, Cambridge/MA: Belknap.

Schrader, U. (2007): The moral responsibility of consumers as citizens, in: International Journal of Innovative and Sustainable Development, Vol. 2/No. 2, 79-96.

Sen, A. (2010): Die Idee der Gerechtigkeit, München: C.H. Beck.

Stehr, N. (2007): Die Moralisierung der Märkte: Eine Gesellschaftstheorie, Frankfurt a. M.: Suhrkamp.

Stoecker, R. (2007): Das Pilatus-Problem und die Vorzüge eines dynamischen Verantwortungsbegriffs, in: Berendes, J. (Hrsg.): Autonomie durch Verantwortung, Paderborn: Mentis, 147-160.

Tetens, H. (2011): Argumentationsstrukturen in der Angewandten Ethik, in: Stoecker, R./Neuhäuser, Ch./Raters M.-L. (Hrsg.): Handbuch Angewandte Ethik, Stuttgart: J.B. Metzler, 18-22.

Ullrich, W. (2007): Habenwollen: Wie funktioniert die Konsumkultur? Frankfurt a. M.: Fischer. Ulrich, P. (1997): Integrative Wirtschaftsethik, Bern: Haupt.

von Alemann, U./Eckert, F. (2006): Lobbyismus als Schattenpolitik, in: Aus Politik und Zeitgeschichte, No. 15-16/2006.

Waldron, J. (1999): Law and Disagreement, Oxford: Oxford University Press.

Wallace, J. (2006): Normativity and the Will, Oxford: Oxford University Press.

Wallace, J. (2011): Konzeptionen der Normativität: Einige grundlegende philosophische Fragen, in: Forst, R./Günther, K. (Hrsg.): Die Herausbildung normativer Ordnungen: Interdisziplinäre Perspektiven, Frankfurt a. M./New York: Campus, 33-56.

Weber, M. (1988): Politik als Beruf, in: Weber, M. (Hrsg.): Gesammelte Politische Schriften, Tübingen: Mohr Siebeck, 505-560.

Young, I. M. (1990): Justice and the Politics of Difference, Princeton: Princeton University Press.

Young, I. M. (2000): Inclusion and Democracy, Oxford: Oxford University Press.

Young, I. M. (2006): Responsibility and Global Justice: A Social Connection Model, in: Social Philosophy and Polity, Vol. 23/No. 1, 102-130.

Young, I. M. (2011): Responsibility for Justice, Oxford: Oxford University Press. 